\title{
Universal Limits on Massless High-Spin Particles
}

\author{
M. Porrati \\ Center for Cosmology and Particle Physics \\ Department of Physics \\ New York University \\ 4 Washington $\mathrm{Pl}$. \\ New York, NY 10003, USA
}

\begin{abstract}
We present a model-independent argument showing that massless particles interacting with gravity in a Minkowski background space can have at most spin two. This result is proven by extending a famous theorem due to Weinberg and Witten to theories that do not possess a gauge-invariant stress-energy tensor.
\end{abstract}




\section{Introduction}

The ever-so-many vacua of string theory [1] differ in almost any conceivable way from one another, yet they all have something in common: none of them describes a fourdimensional Minkowski space with massless particles of spin larger than two. The graviton is always the highest-spin massless state. Massive particles of any spin do exist, but they are always composite states, or unstable resonances. More precisely, their mass $M_{s>2}$ is always larger than the string scale $M_{S}$, and they are not point-like: they possess form factors that give them a size $L \gtrsim 1 / M_{S} \gtrsim 1 / M_{s>2}$. Hadronic resonances also have a finite, nonzero size $L \sim 1 / M$; classical spinning objects of course have $L \gg 1 / M$.

We take these facts as hints that high-spin particles become strongly interacting at a finite energy scale $\Lambda$. How is $\Lambda$ related to the particle mass $M$ and its spin $s$ and how does the strong coupling regime manifests? In string theory we can give a concrete answer to this question: a multitude of other states of spin less than $s$ exists at or below the mass $M$. Their multiplicity is exponential in $M: D(M) \sim \exp \left(c M / M_{S}\right)$. (c is a numerical constant that depends on the specific string theory being considered.) The limit $M_{S} \rightarrow 0$ is singular: it produces an infinite number of massless states, whose interactions have not yet been properly understood.

No example exists of a string compactification with a light high-spin state, i.e. an $s>2$ state with mass $M_{s>2} \ll M_{S}$. More generally, no theory is known where particles of spin $s>2$ and mass $M_{s>2}$ interact weakly up to an arbitrarily high energy scale. In all known examples, regardless of the precise functional relation linking $\Lambda$ to $M_{s>2}, \Lambda$ vanishes in the massless limit $M_{s>2} \rightarrow 0$.

What we said applies to flat Minkowski backgrounds. In Anti de Sitter space, theories with infinitely many massless particles of arbitrary spin are known [2]. Their spectrum cannot be consistently truncated to a finite number of particles. Even more importantly, their interactions scale as inverse powers of the cosmological constant $\lambda$, so the flat space limit $\lambda \rightarrow 0$ is singular. In AdS the cosmological constant plays the role of the mass scale which determines the onset of the strong coupling regime. Thus, far from being in contradiction with our previous flat-space examples, AdS models point out to the same conclusion: when the relevant mass scale of our theory goes to zero, be it $M$ or $\sqrt{|\lambda|}$, a high-spin particle becomes strongly interacting. In formulas, if we denote by $\tilde{M}$ the largest between $M$ and $\sqrt{|\lambda|}$, the effective coupling of the theory at an energy scale $E$ behaves as

$$
g_{e f f}(E) \sim \frac{E^{a+b}}{M_{*}^{a} \tilde{M}^{b}}, \quad a, b>0 .
$$

To be general, we introduced another mass scale, $M_{*}$, which characterizes possible in- 
teractions of the high-spin state with either itself or other particles. Since all particles should interact at least with gravity, a universal choice for $M_{*}$ is the Planck mass $M_{P l}$.

Equation (11) is motivated by a simple observation: if a high-spin theory had a well defined $M_{s>2} \rightarrow 0$ limit, then it would be possible to construct massless high-spin theories interacting at least with gravity. Yet, strong constraints exist in the literature, that forbid this possibility. All known no go arguments or theorems have loopholes; aim of this paper is to close some of those loopholes.

We will review the main existing no go theorems on interacting high-spin theories in Section 2; in particular, we will briefly re-derive the Weinberg-Witten theorem [3]. By a suitable weakening of its hypotheses, it will give us the desired no gd, presented in Section 3. Specifically, Section 3 presents our argument, showing that particles of spin larger than two cannot have gravitational interactions in Minkowski space. The proof parallels the seminal results obtained in a Lagrangian framework by Aragone and Deser for spin 5/2 [4]; it extends their results beyond their local field theory framework, and it generalizes it to arbitrary spins. Section 4 contains a discussion of our result, its limitations and possible extensions, as well as an application of the methods of Section 3 to the simpler case of charged particles in interaction with massless Abelian gauge fields. Section 4 also puts forward some speculations on how to circumvent our no go theorem.

\section{A Brief History of No Go Theorems}

An important obstruction to consistent interactions of high-spin massless particles was derived in 1964 by Weinberg [5] using general properties of the S-matrix. His result was extended to Fermions and specifically to supersymmetric theories in [6, 7].

We shall review now Weinberg's result since we will use later one of its key techniques. Consider an S-matrix element with $N$ external particles of four-momentum $p_{i}, i=1, . . N$ and one massless spin $s$ particle of momentum $q$ and polarization vector $\epsilon^{\mu_{1} . . \mu_{s}}(q)$. In the soft limit $q \rightarrow 0$, it factorizes as (see fig. 1)

$$
S\left(p_{1}, . ., p_{N}, q, \epsilon\right) \approx \sum_{i=1}^{N} g^{i} \frac{p_{\mu_{1}}^{i} \ldots p_{\mu_{s}}^{i} \epsilon^{\mu_{1} . . \mu_{s}}(q)}{2 p^{i} q} S\left(p_{1} \ldots p_{N}\right)
$$

\footnotetext{
${ }^{1}$ As for all no go theorems, ours should be rather called don't go there. These theorems often allow exceptions, and their constructive role is precisely to show which avenue one should not take in the search for self-consistent theories.
} 
The polarization vector is transverse and traceless

$$
q_{\mu} \epsilon^{\mu \mu_{2} . . \mu_{s}}(q)=0, \quad \epsilon_{\mu}^{\mu \mu_{3} . . \mu_{s}}(q)=0 .
$$

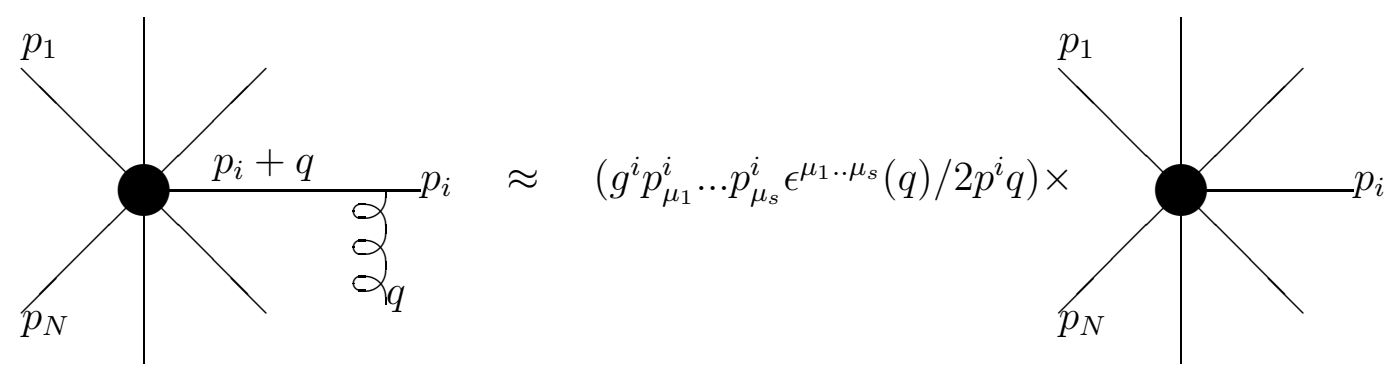

Figure 1: Factorization of S-matrix amplitude in the soft limit $q \rightarrow 0$.

It gives a redundant description of the massless particle, which has only two physical polarizations. Redundancy is eliminated by demanding that the S-matrix is independent of spurious polarizations

$$
\epsilon_{\text {spurious }}^{\mu_{1} \ldots \mu_{s}}(q) \equiv q^{\left(\mu_{1}\right.} \eta^{\left.\mu_{2} . . \mu_{s}\right)}(q), \quad q_{\mu} \eta^{\mu \mu_{1} . . \mu_{s-2}}(q)=\eta_{\mu}^{\mu \mu_{1} . . \mu_{s-3}}(q)=0 .
$$

Factorization eq. (2) implies that spurious polarizations decouple only when

$$
\sum_{i} g^{i} p_{\mu_{1}}^{i} \ldots p_{\mu_{s-1}}^{i}=0, \quad \forall p_{i}
$$

For generic momenta this equation has a solution only in two cases:

$s=1$ In this case eq. (5) reduces to $\sum_{i} g^{i}=0$, i.e. to conservation of charge.

$s=2$ Eq. (5) becomes $\sum_{i} p_{\mu}^{i}=0$ and $g^{i}=\kappa$. The first equation enforces energymomentum conservation, while the second gives the principle of equivalence: all particles must interact with the massless spin two with equal strength $\kappa$.

For $s>2$ eq. (5) has no solution for generic momenta.

This argument shows that only scalars, vectors and spin two particles interacting at long distance as gravitons can give rise to long-distance interactions. The argument was extended to Fermions in [6, 7], where it was shown that interacting massless Fermions exist only up to spin 3/22. Both [5] and [6, 7] rely on the existence of processes in which

\footnotetext{
${ }^{2}$ Spin $3 / 2$ Fermions were also shown to interact as the supersymmetric partners of the graviton, i.e.
} 
the number of spin $s$ particles changes by one unit. This is necessary to generate longrange interactions for integer $s$, but it leaves out the possibility of interacting high-spin particles with a nonzero conserved charge. In particular, particles interacting only with the graviton according to the principle of equivalence are still allowed. Moreover, the interaction of these particles could be softened by powers of $q_{\mu}$ in such a manner as to cancel the offending pole in eq. (22). These particles do not generate long range forces, but they can still interact.

If we want to exclude completely high-spin massless particles, we must look for a truly universal interaction, one that no particle can avoid. The best choice is the gravitational interaction. Equation (5) shows that the graviton interacts universally with matter in the soft limit $q \rightarrow 0$. Indeed, eq. (5) can be taken as the most general form of the equivalence principle: all matter interacts with the graviton and in the limit $q \rightarrow 0$ the interaction vertex is $\kappa\left\langle f\left|T_{\mu \nu}\right| i\right\rangle(|i\rangle,|f\rangle$ are the particle's initial and final states, respectively).

Inconsistencies of gravitationally coupled high-spin massless particles were specifically studied in [4] for $s=5 / 2$. It is instructive to review the argument presented there, since we will extend some of its techniques to a more general S-matrix framework in Section 4. Ref. [4] writes down a local field theory for a spin 5/2 field, described by a tensor-spinor $\psi_{a b}$, coupled to gravity, described by the tetrad $e_{a}^{\mu}$. To quadratic order in $\psi_{a b}$ it reads

$$
S=\int d^{4} x e\left[-\frac{1}{2} \bar{\psi}_{a b} \not D \psi_{a b}-\bar{\psi}_{a b} \gamma_{b} \not D \gamma_{c} \psi_{c a}+2 \bar{\psi}_{a b} \gamma_{b} D_{c} \psi_{c a}+\frac{1}{4} \bar{\psi}_{a a} \not D \psi_{b b}-\bar{\psi}_{a a} D_{b} \gamma_{c} \psi_{b c}\right]
$$

The field $\psi_{a b}$ gives a redundant description of the spin $5 / 2$ state. In the free theory this redundancy is eliminated by the gauge invariance

$$
\delta \psi_{\mu \nu}=\partial_{\mu} \epsilon_{\nu}+\partial_{\nu} \epsilon_{\mu}, \quad \gamma^{\mu} \epsilon_{\mu}=0
$$

Upon covariantization, derivatives are replaced by covariant derivatives, but the gauge transformation is otherwise unaffected

$$
\delta \psi_{a b}=D_{a} \epsilon_{b}+D_{b} \epsilon_{a}, \quad \gamma^{a} \epsilon_{a}=0
$$

Under the gauge transformation (8) action (6) transforms as

$$
\delta S=-4 \int d^{4} x e \bar{\epsilon}_{a} \gamma_{b} \psi_{c d} R^{a b c d}
$$

So, the action is only invariant in flat space $R^{a b c d}=0$; in other words, gauge modes the gravitini of supergravity theory. 
decouple only in the free theory. It is quite immediate to convince oneself that this inconsistency cannot be cured by adding non-minimal terms to action (6) that are both local and regular in the neighborhood of flat space. If the last requirement is removed, as it is possible in a theory that makes sense e.g. in Anti de Sitter space, but which does not allow for a flat space limit, then we can not only decouple the gauge modes, but also write down a consistent theory of high-spin massless Fermions. To achieve consistency beyond the lowest perturbative order, one must nevertheless introduce (infinitely many) new massless states besides the spin $5 / 2$ on 3 .

The cosmological constant $\lambda<0$ appearing in AdS space defines a mass parameter $O(\sqrt{|\lambda|})$. Massive spin $5 / 2$ also evades rather trivially the no-go, since the gauge invariance is already broken by the mass term present in the massive analog of eq. (66). Interacting, massive high-spin theories are not algebraically inconsistent, but they do manifest pathologies ranging from superluminal propagation and ghosts in external coherent fields [8] to strong coupling behavior at a finite energy scale [9, 10, 11]. In fact, in AdS space one should expect no operational difference between massless particles and particles with Compton wavelength $\lambda_{\text {Compton }} \sim 1 / m$ larger than the AdS curvature radius $R_{A d S} \sim 1 / \lambda$. This expectation is confirmed by the absence of mass discontinuities in their propagators [12, 13, 14, 15].

Returning now to massless particles in asymptotically Minkowski space, we could imagine that the inconsistency evidenced by eq. (9) is due to the most important implicit assumption inherent to the Lagrangian formalism: locality. Eqs. (6) and ff assume a standard kinetic term for $\psi_{a b}$, and local interactions. Could it be that a carefully chosen form factor for gravitational interactions, tantamount to some reasonable non-locality in the Lagrangian can cure the problem? How does our result depend on the field representation chosen for the spin 5/2 particle? Could a non-minimal description, involving a larger gauge invariance than that in eqs. (7,8) be consistent after all? To answer all these questions, we must use a truly universal formalism, dealing with matrix elements of observables. In other words, we should go back to the S-matrix language and use it to analyze an unavoidable interaction: scattering of massless particles off soft gravitons.

This analysis was done in [3], where a particular matrix element was considered: elastic scattering of a spin $s$ massless particle off a single soft graviton. The initial and final polarizations of the spin $s$ particle are identical, say $+s$, its initial momentum is $p$ and its final momentum is $p+q$. The graviton is off-shell with momentum $q$. The matrix

\footnotetext{
${ }^{3}$ Literature on this subject is vast and complex. Comprehensive reviews of (Bosonic) high-spin theories in AdS, with extended bibliography, can be found in [2].
} 
element is

$$
\left\langle+s, p+q\left|T_{\mu \nu}\right|+s, p\right\rangle \text {. }
$$

In the soft limit $q \rightarrow 0$ the matrix element is completely determined by the equivalence principle. Using the relativistic normalization for one-particle states, $\left\langle p \mid p^{\prime}\right\rangle=$ $2 p_{0}(2 \pi)^{3} \delta^{3}\left(\mathbf{p}-\mathbf{p}^{\prime}\right)$, we get

$$
\lim _{q \rightarrow 0}\left\langle+s, p+q\left|T_{\mu \nu}\right|+s, p\right\rangle=p_{\mu} p_{\nu}
$$

Since $q$ is space-like, there exists a frame -the "brick wall" frame- in which

$$
p^{\mu}=(|\mathbf{q}| / 2, \mathbf{q} / 2), \quad q^{\mu}=(0,-\mathbf{q}), \quad p^{\mu}+q^{\mu}=(|\mathbf{q}| / 2,-\mathbf{q} / 2) .
$$

A rotation $R(\theta)$ by an angle $\theta$ around the $\mathbf{q}$ direction acts on the one-particle states as

$$
R(\theta)|p,+s\rangle=\exp ( \pm i \theta s)|p,+s\rangle, \quad R(\theta)|p+q,+s\rangle=\exp (\mp i \theta s)|p+q,+s\rangle,
$$

since $R(\theta)$ is a rotation of $\theta$ around $\mathbf{p}$ but of $-\theta$ around $\mathbf{p}+\mathbf{q}=-\mathbf{p}$. Under space rotations, $T_{\mu \nu}$ decomposes into two real scalars, one vector and one symmetric traceless tensor. In the standard basis where the commuting variables are the total angular momentum and its projection along the axis $\mathbf{q}$, these field are represented by spherical tensors: $T_{0,0}, T_{1, m}$, $m=0, \pm 1$ and $T_{2, m}, m=0, \pm 1, \pm 2$. Here we have combined the two real scalars into a complex scalar. In this basis one gets the trivial identity

$$
e^{ \pm 2 i \theta s}\left\langle+s, p+q\left|T_{j, m}\right|+s, p\right\rangle=\left\langle+s, p+q\left|R^{\dagger} T_{j, m} R\right|+s, p\right\rangle=e^{i \theta m}\left\langle+s, p+q\left|T_{j, m}\right|+s, p\right\rangle .
$$

For $s>1$, the only solution to this equation is $\left\langle+s, p+q\left|T_{\mu \nu}\right|+s, p\right\rangle=0$.

If $T_{\mu \nu}$ is a tensor under Lorentz transformations then eq. (14) implies that the matrix element (10) vanishes in all frames, in contradiction with the equivalence principle eq. (11)!

The crucial assumption here is that $T_{\mu \nu}$ (better, its matrix element between massless spin $s$ states) is a Lorentz tensor. The assumption is far from innocuous. In particular, neither the gravitino ( $\operatorname{spin} 3 / 2$ ) nor the graviton (spin 2) satisfy this hypothesis [3]. This happens because both spin $3 / 2$ and spin 2 have gauge invariances (local supersymmetry and diffeomorphisms, respectively) and their stress-energy tensor is not gauge invariant. The stress-energy tensor derived from Lagrangian higher-spin theories exhibits the same phenomenon: to achieve gauge invariance one must forgo manifest Lorentz covariance [16].

In fact, non-Lorentz covariance of gauge non-invariant operators is a familiar fact in field theory. The simplest example is offered by the matrix element of the EM gauge 
potential $A_{\mu}$ in between the vacuum and a one-photon state, $\left\langle 0\left|A_{\mu}\right| s, p\right\rangle(s= \pm 1)$. One can always choose a complete set of polarization vectors for $A_{\mu}$ such that $\left\langle 0\left|A_{0}\right| s, p\right\rangle=0$. A Lorentz boost $L$ leaves the vacuum invariant and transforms the one particle state as $L|s, p\rangle=\exp [i \theta(L, p) s]|s, p\rangle$; therefore, $\left\langle 0\left|L^{\dagger} A_{0} L\right| s, p\right\rangle=\exp [i \theta(L, p) s]\left\langle 0\left|A_{0}\right| s, p\right\rangle=0$ in all frames. This is of course incompatible with the transformation law of a Lorentz vector. Indeed a Lorentz boost transforms inhomogeneously the matrix element. In infinitesimal form, the transformation law is a standard Lorentz transformation plus a compensating gauge transformation

$$
\delta_{\omega}\left\langle 0\left|A_{\mu}\right| s, p\right\rangle=\omega_{\mu}^{\nu}\left\langle 0\left|A_{\nu}\right| s, p\right\rangle+p_{\mu} \Phi(p, \omega)
$$

One can define matrix elements for $A_{\mu}$ that transform as Lorentz vectors, but only at the price of introducing non-physical states which correspond to spurious polarizations. Likewise, one can define $T_{\mu \nu}$ matrix elements that transform as Lorentz tensors by introducing spurious polarizations. These facts are the crucial ingredient in our treatment of high-spin massless fields.

\section{An Improved No Go Theorem}

To replace eq. (10) with a Lorentz covariant matrix element we need extra spurious states, besides the physical ones given in eq. (13). These states mix with physical polarizations under Lorentz transformations: $v_{\text {phys }}-->v_{\text {phys }}+v_{\mathrm{s}} 4$. Physical and spurious states together thus span a reducible but not block-diagonal representation of the Lorentz group. Spurious states must decouple from all physical matrix elements and in particular from S-matrix amplitudes. If we denote with $v$ all one-particle, spin $s$ states, whether or not spurious, the matrix element is $\left\langle v^{\prime}, p+q\left|T_{\mu \nu}\right| v, p\right\rangle$. It is not an S-matrix element yet, since the graviton (and only the graviton) is off-shell. A convenient method to derive the S-matrix is to perform the standard perturbative expansion of the effective action

$$
A=\frac{1}{16 \pi G} \int d^{4} x \sqrt{-g} R(g)+\frac{1}{2} \int \frac{d^{4} q}{(2 \pi)^{4}} h_{\mu \nu}^{*}(q)\left[\left\langle v^{\prime}, p+q\left|T^{\mu \nu}\right| v, p\right\rangle+\mathcal{T}^{\mu \nu}\right]+\mathcal{O}\left(h^{2}\right)
$$

The standard Einstein action with Newton's constant $G$ and metric $g_{\mu \nu}$ has been supplemented here with certain interaction terms, written in a perturbative expansion around flat space $\left(g_{\mu \nu}=\eta_{\mu \nu}+h_{\mu \nu}\right)$. The linear interaction terms include our matrix element and another effective stress energy tensor $\mathcal{T}_{\mu \nu}$, which summarizes the effect of any other

\footnotetext{
${ }^{4}$ We saw this phenomenon at work in the case of spin one in eq. (15).
} 
matter field. Eq. (16) is not only a convenient bookkeeping device, but it also gives us the most general condition for the decoupling of a spurious polarization $v_{s}$. Decoupling occurs when one can reabsorb the change in the matrix element due to the substitution $v \rightarrow v+v_{s}$ with a local field redefinition of the graviton field $h_{\mu \nu}$. This happens because the S-matrix is independent of such redefinition [17]. To linear order in $h_{\mu \nu}$, Einstein's equations become

$$
\begin{aligned}
L_{\mu \nu}{ }^{\rho \sigma} h_{\rho \sigma}(q) & =16 \pi G\left[\left\langle v^{\prime}, p+q\left|T_{\mu \nu}\right| v, p\right\rangle+\mathcal{T}_{\mu \nu}\right] \\
L_{\mu \nu}{ }^{\rho \sigma} & =\delta_{\mu \nu}^{\rho \sigma} q^{2}-\delta_{\mu \nu} \delta^{\rho \sigma} q^{2}-\delta_{\mu}^{\rho} q_{\nu} q^{\sigma}-\delta_{\nu}^{\rho} q_{\mu} q^{\sigma}+\delta^{\rho \sigma} q_{\mu} q_{\nu}+\delta_{\mu \nu} q^{\rho} q^{\sigma}
\end{aligned}
$$

To the same order, we then get a necessary condition for the consistency of gravitational interactions of high-spin massless particles:

$$
\left\langle v, p+q\left|T_{\mu \nu}\right| v_{s}, p\right\rangle=L_{\mu \nu}^{\rho \sigma} \Delta_{\rho \sigma}(q)
$$

with $\Delta_{\mu \nu}(q)$ analytic in a neighborhood of $q=0$. The required field redefinition is $h_{\mu \nu} \rightarrow h_{\mu \nu}+16 \pi \Delta_{\mu \nu}(q)$.

Eq. (18) weakens the hypotheses of the Weinberg-Witten theorem by allowing the matrix element to depend non-trivially on spurious polarizations. In Lagrangian language, this means that the stress-energy is not separately gauge invariant, though the action is. The Weinberg-Witten result is recovered by demanding the stronger condition $\Delta_{\mu \nu}(q)=0$, i.e. gauge invariance.

Eq. (18) does not guarantee the existence of a consistent theory, since inconsistencies can show up in contact terms at $\mathcal{O}\left(h^{2}\right)$, but if not satisfied it signals a lethal inconsistency, since in that case no amount of extra fields or extra interactions can cancel the $v_{s}$ dependent change in the action. Notice that while $\Delta_{\mu \nu}(q)$ must be analytic in $q$ for small $q$, no such requirement holds for the matrix element itself. This is a first advantage of the S-matrix formalism over the Lagrangian analysis of ref. 4], which we summarized in Section 2. In a Lagrangian framework one must necessarily assume locality of the matrix element itself; moreover, one is still left with the doubt that a field redefinition of $\psi_{\mu \nu}$ may change the analysis. In our case, since the initial and final spin $s$ states are on-shell, no such redefinition exists.

The last observation also answers another question about the generality of our result: can it depend on the particular choice of spurious states we are going to make? No, it can't. A non-minimal choice of spurious states means to introduce a larger set of them, which we can denote with $\left\{V_{s}\right\}$. By setting some of them to zero, we go back to our 
minimal choice (to be defined shortly), $\left\{v_{s}\right\} \subset\left\{V_{s}\right\}$. Independence of $\left\{V_{s}\right\}$ thus implies independence of all $v_{s}$, which implies eq. (18).

\subsection{Fermions}

The matrix element $\left\langle v^{\prime}, p+q\left|T_{\mu \nu}\right| v, p\right\rangle$ is bilinear in $v, v^{\prime}$ and it otherwise depends only on the momenta. For spin $s$, the minimum set of spurious states needed to write a nonzero conserved, symmetric tensor is given by Dirac spinor-tensors $v_{\alpha, \mu_{1} \ldots \mu_{n}}(p), s=n+1 / 2$. They are symmetric in the vector indices $\mu_{1},, \mu_{n}$ and satisfy the constraints

$$
\not p v_{\mu_{1}, . . \mu_{n}}(p)=0, \quad p^{\mu_{1}} v_{\mu_{1}, . . \mu_{n}}(p)=0, \quad \gamma^{\mu_{1}} v_{\mu_{1}, . . \mu_{n}}(p)
$$

We are interested in initial and final states with the same physical helicity $+s$, so on the representatives of the initial state $(u)$ and final state $(v)$ we impose

$$
\gamma^{5} u_{\mu_{1}, . . \mu_{n}}(p)=u_{\mu_{1}, . . \mu_{n}}(p), \quad \gamma^{5} v_{\mu_{1}, . . \mu_{n}}(p+q)=v_{\mu_{1}, . . \mu_{n}}(p+q) .
$$

In the kinematical configuration of interest, there exist two independent light-like vectors: $p$ and $p+q$. The space-like vector $q$ can be used to define $n+1$ algebraically independent spinor-tensors

$$
u_{\mu_{1}, . ., \mu_{k}}^{k}(p) \equiv q^{\mu_{k+1}} \ldots q^{\mu_{n}} u_{\mu_{1}, . . \mu_{n}}(p), \quad k=0, . ., n
$$

Their algebraic independence is verified by writing down their explicit parametrization in the brick wall frame eq. (12). Introduce first of all vector polarizations $\epsilon$ and an on-shell spinor $\chi$

$$
\epsilon_{\mu}^{3}=(-1,1,0,0), \quad \epsilon_{\mu}^{ \pm}=(0,0,1, \pm i), \quad \gamma^{5} \chi=\chi, \quad\left(\gamma^{0}-\gamma^{1}\right) \chi=0
$$

The last equation is the on-shell condition $\not p \chi(p)=0$; the last two conditions imply $\left(\gamma^{2}+i \gamma^{3}\right) \chi=0$. The $n+1$ spinor tensors

$$
u_{\mu_{1}, . . \mu_{n}}^{(k)} \equiv \epsilon_{\left(\mu_{1}\right.}^{+} \ldots \epsilon_{\mu_{k}}^{+} \epsilon_{\mu_{k+1}}^{3} \epsilon_{\left.\mu_{n}\right)}^{3} \chi, \quad k=0, . ., n
$$

are evidently linearly independent, they satisfy the constraints (19,201) and obey

$$
u_{\mu_{1}, . . \mu_{l}}^{(k) l} \equiv q^{\mu_{l+1}} \ldots q^{\mu_{n}} u_{\mu_{1}, . . \mu_{n}}^{(k)} \begin{cases}=0, & \text { for } l<k \\ \neq 0, & \text { for } l \geq k\end{cases}
$$


The triangular linear system (24) defines $n+1$ independent spinor tensors. Eq. (21) or (24) parametrize one physical polarization of helicity $s=n+1 / 2$ and $n$ spurious polarizations with $s=1 / 2, \ldots, n-1 / 25$.

Constraints (19,20) and the on-shell condition on momenta, $p^{2}=(p+q)^{2}=0$, vastly reduce the possible terms in the matrix element of interest. A short reflection suffices to convince oneself that its most general form is

$$
\left\langle v, p+q\left|T_{\mu \nu}\right| u, p\right\rangle=\sum_{k=0}^{n} A^{k} \bar{v}^{k}\left(p+\alpha^{k} q\right)_{(\mu} \gamma_{\nu)} u^{k}+\sum_{k=1}^{n} B^{k} \bar{v}_{(\mu}^{k} \gamma_{\nu)} u^{k-1}+\sum_{k=1}^{n} C^{k} \bar{v}_{(\mu}^{k-1} \gamma_{\nu)} u^{k}
$$

The coefficients $A^{k}, B^{k}, C^{k}$ and $\alpha^{k}$ are functions of $q^{2}$ which in principle can be singular at $q^{2}=0$. A first constraint on the singularity is due to the principle of equivalence that demands

$$
\lim _{q \rightarrow 0}\left\langle v, p+q\left|T_{\mu \nu}\right| u, p\right\rangle=p_{\mu} p_{\nu}
$$

This equation implies

$$
\begin{aligned}
\lim _{q \rightarrow 0} A^{n}(q) & =1, \\
\lim _{q \rightarrow 0} A^{k}(q) q^{2(n-k)} & =0, \quad k<n, \\
\lim _{q \rightarrow 0} \alpha^{k}(q) A^{k}(q) q^{2(n-k)-1} & =0, \\
\lim _{q \rightarrow 0} B^{k}(q) q^{2(n-k)+1} & =0, \\
\lim _{q \rightarrow 0} C^{k}(q) q^{2(n-k)+1} & =0 .
\end{aligned}
$$

Conservation of $T_{\mu \nu}$ implies that the matrix element (25) is divergenceless

$$
q^{\mu}\left\langle v, p+q\left|T_{\mu \nu}\right| u, p\right\rangle=0
$$

This yields the further constraints

$$
\begin{aligned}
A^{k}\left(\alpha^{k}-1 / 2\right) q^{2}+B^{k+1}+C^{k+1} & =0, \quad k=0, . ., n-1, \\
\lim _{q \rightarrow 0} \alpha^{n}(q) & =1 / 2, \quad \lim _{q \rightarrow 0} A^{n}(q)=1 .
\end{aligned}
$$

Though not strictly necessary to prove our result, eq. (34) is useful since it simplifies the structure of the matrix element. In particular, together with the mass-shell conditions (19) it makes the matrix element transverse and traceless.

\footnotetext{
${ }^{5} s<0$ states are eliminated by the chirality projection $\gamma^{5} u_{\mu_{1}, . . \mu_{n}}=u_{\mu_{1}, . . \mu_{n}}$.
} 
In reality, constraints (27,31) are too weak, because if any of the coefficients $A^{k}, B^{k}, C^{k}$ and $\alpha^{k} A^{k}$ had a singularity $1 / q^{2} 6$ then vertex (25) would imply the existence of another massless spin 2 field (it couples to a transverse-traceless vertex!) which mixes linearly with the graviton. This linear mixing contradicts Weinberg's uniqueness theorems for soft gravitons [18]. It also violates the principle of equivalence - which we assumed (and need) to prove or theorem- either because it implies the existence of a second massless graviton that couples only to some type of matter (massless high-spin) or because it re-sums to give the graviton a mass. A singularity stronger than $1 / q^{2}$ is even worse since it implies the existence of a spin two ghost mixing linearly with the ordinary graviton (see fig. 2).

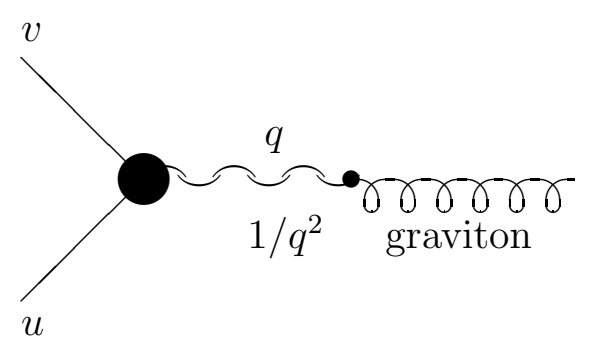

Figure 2: A singular vertex implies the existence of an additional massless particle mixing with the graviton.

We have introduced extra polarizations to ensure that the $T_{\mu \nu}$ matrix element transforms covariantly. Now we must check under which conditions spurious polarizations do decouple. Spurious states have the form

$$
u_{s \mu_{1} \ldots \mu_{n}}(p)=p_{\left(\mu_{1}\right.} \epsilon_{\left.\mu_{2} \ldots \mu_{n}\right)},
$$

where $\epsilon_{\mu_{1} \ldots \mu_{n-1}}$ is on shell, transverse and gamma-transverse. For the spurious state (35), the spinor-tensors given in eq. (21) have the form

$$
u_{s \mu_{1} \ldots \mu_{k}}^{k}(p)=p_{\left(\mu_{1}\right.} \epsilon_{\left.\mu_{2} \ldots \mu_{k}\right)}^{k-1}-(n-k) \frac{q^{2}}{2} \epsilon_{\mu_{1} \ldots \mu_{k}}^{k}, \quad \epsilon_{\mu_{1} \ldots \mu_{k}}^{k} \equiv q^{\mu_{k+1} \ldots q^{\mu_{n}}} \epsilon_{\mu_{1} \ldots \mu_{n}}^{k}
$$

Matrix element (25) is transverse and traceless, therefore the decoupling condition (18) simplifies to

$$
\left\langle v, p+q\left|T_{\mu \nu}\right| u_{s}, p\right\rangle=q^{2} \Delta_{\rho \sigma}(q) .
$$

\footnotetext{
${ }^{6}$ For instance $A^{k}(q)=A_{r}^{k}(q) q^{-2}, A_{r}^{k}(q)=$ regular and nonzero at $q^{2}=0$.
} 
Substitution of eqs. (35, 36) into eq. (25) then yields a set of recursion relations among the coefficients $A^{k}, \ldots, C^{k}$ :

$$
\begin{aligned}
& -k A^{k}-\frac{q^{2}}{2}(n+1-k) A^{k-1}+C^{k}=\mathcal{O}\left(q^{2}\right), \quad k=1, \ldots, n \\
& -k \alpha^{k} A^{k}-\frac{q^{2}}{2}(n+1-k) \alpha^{k} A^{k-1}=\mathcal{O}\left(q^{2}\right), \quad k=1, \ldots, n ; \\
& -(k-1) B^{k}-\frac{q^{2}}{2}(k+2-k) B^{k-1}=\mathcal{O}\left(q^{2}\right), \quad k=2, \ldots, n ; \\
& -(k-1) C^{k}-\frac{q^{2}}{2}(k+1-k) C^{k-1}=\mathcal{O}\left(q^{2}\right), \quad k=2, \ldots, n .
\end{aligned}
$$

As we have seen earlier, no coefficient in eq. (25) can be more singular than $1 / q^{2}$. So in particular

$$
\lim _{q \rightarrow 0} q^{2} C^{1}(q)=\lim _{q \rightarrow 0} q^{2} A^{0}(q)=0 .
$$

Recursion relations (38, 41) then imply

$$
\lim _{q \rightarrow 0} A^{n}(q)=0, \quad n>1
$$

in contradiction with eq. (27), $A^{n}(0)=1$, which is nothing else than the equivalence principle!

This completes our proof: only when spurious polarizations decouple from the cubic vertex (25) a chance exists for massless high-spin fields to interact with gravity, but decoupling contradicts the universality of gravitational interactions!

Our argument rules out interactions for Fermions of spin $s>3 / 2$. It still allows for gravitational interactions of spin $3 / 2$ particles. This is not surprising since supergravity theories provide many examples of massless spin $3 / 2$ particles consistently interacting with gravity and other fields.

Notice that our argument does not rule out exotic high-spin interacting theories, but it shows that these theories do not have any common interaction with physical matter, which must interact with gravity universally.

Notice too that our argument relies crucially on the exact masslessness of the graviton. In the conclusions, we will briefly discuss the dynamics of high-spin massless particles in theories where gravity changes in the infrared, as in massive gravity or in the DGP model [19]. 


\subsection{Bosons}

The proof of our theorem in the Bosonic case parallels that we gave for Fermions. Polarizations are now described by the on-shell, symmetric, transverse and traceless tensors

$$
U_{\mu_{1} . . \mu_{s}}(p), \quad p^{\mu_{1}} U_{\mu_{1 . . .} \mu_{s}}(p)=U_{\mu_{2} . . \mu_{s}}^{\mu_{2}}(p)=0
$$

Spurious polarizations read

$$
U_{\mu_{1} . . \mu_{s}}(p)=p_{\left(\mu_{1}\right.} \epsilon_{\left.\mu_{2} \ldots \mu_{s}\right)}, \quad p^{\mu_{2}} \epsilon_{\mu_{2} \ldots \mu_{s}}=\epsilon_{\mu_{3} \ldots \mu_{s}}^{\mu_{3}}=0
$$

In complete analogy with the Fermion treatment, we use contraction with $q^{\mu}$ to define

$$
U_{\mu_{1} \ldots \mu_{k}}^{k}(p)=q^{\mu_{k+1} \ldots} q^{\mu_{s}} U_{\mu_{1} \ldots \mu_{s}}(p), \quad \epsilon_{\mu_{1} \ldots \mu_{k}}^{k}=q^{\mu_{k+1} \ldots} q^{\mu_{s-1}} \epsilon_{\mu_{1} \ldots \mu_{s-1}}
$$

Contraction of the spurious polarizations defined by eq. (45) results in

$$
U_{\mu_{1} . . \mu_{k}}^{k}(p)=k p_{\left(\mu_{1}\right.} \epsilon_{\left.\mu_{2} \ldots \mu_{k}\right)}^{k-1}-(s-k) \frac{q^{2}}{2} \epsilon_{\mu_{1} \ldots \mu_{k}}^{k}
$$

The most general form of the matrix element $\left\langle V, p+q\left|T_{\mu \nu}\right| U, p\right\rangle$ is now

$$
\begin{aligned}
\left\langle V, p+q\left|T_{\mu \nu}\right| U, p\right\rangle= & \sum_{k=0}^{s} \frac{A^{k}}{2}\left(p_{\mu} p_{\nu}+2 \alpha^{k} p_{\mu} q_{\nu}+\tilde{\alpha}^{k} q_{\mu} q_{\nu}+\hat{\alpha}^{k} \eta_{\mu \nu}\right) \bar{V}^{k} U^{k}+ \\
& \sum_{k=0}^{s-1} B^{k}\left(p_{\nu}+\beta^{k} q_{\nu}\right) \bar{V}_{\mu}^{k+1} U^{k}+\sum_{k=0}^{s-1} C^{k}\left(p_{\nu}+\gamma^{k} q_{\nu}\right) \bar{V}^{k} U_{\mu}^{k+1}+ \\
& \sum_{k=0}^{s-1} D^{k} \bar{V}_{\mu}^{k+1} U_{\nu}^{k+1}+\sum_{k=0}^{s-2} E^{k} \bar{V}_{\mu \nu}^{k+2} U^{k}+\sum_{k=0}^{s-2} F^{k} \bar{V}^{k} U_{\mu \nu}^{k+2} .
\end{aligned}
$$

By contracting expansion (48) with $q^{\mu}$ and equating all algebraically independent terms to zero, we enforce conservation of the stress-energy tensor. As for Fermions, while not necessary to prove our result, $q^{\mu}$ transversality somewhat simplifies the algebra. Specifically, vanishing of terms proportional to $\bar{V}_{\mu}^{k+1} U^{k}$ yields the equation

$$
B^{k}\left(\beta^{k}-1 / 2\right) q^{2}+D^{k}+E^{k}=0, \quad k \leq s-1,
$$

where we defined $E^{s-1} \equiv 0$. Setting to zero terms proportional to $\bar{V}^{k} U_{\mu}^{k+1}$ we get

$$
C^{k}\left(\gamma^{k}-1 / 2\right)+D^{k}+F^{k}=0, \quad k \leq s-1, \quad F^{s-1} \equiv 0 .
$$


Finally, vanishing of terms proportional to $p_{\mu} \bar{V}^{k} U^{k}$ implies

$$
A^{s}\left(\alpha^{s}-1 / 2\right)=0, \quad A^{k}\left(\alpha^{k}-1 / 2\right) q^{2}+B^{k}+C^{k}=0, \quad k \leq s-1,
$$

while vanishing of terms proportional to $q_{\mu} \bar{V}^{k} U^{k}$ results in

$$
A^{s}\left[\hat{\alpha}^{s}+q^{2}\left(\tilde{\alpha}^{s}-\alpha^{s} / 2\right)\right]=0, \quad A^{k}\left[\hat{\alpha}^{k}+q^{2}\left(\tilde{\alpha}^{k}-\alpha^{k} / 2\right)\right]+B^{k} \beta^{k}+C^{k} \gamma^{k}=0, \quad k \leq s-1 .
$$

Matrix element (48) is traceless for

$$
A^{0} \hat{\alpha}^{0}=B^{0} / 4, \quad A^{s} \hat{\alpha}^{s}=-D^{s-1} / 4, \quad A^{k} \hat{\alpha}^{k}=\left(B^{k}-D^{k-1}\right) / 4, \quad k=1, \ldots, s-1 .
$$

A generic conserved symmetric tensor can be decomposed into a transverse-traceless $(T T)$ part and a scalar remnant as $\Theta_{\mu \nu}=\Theta_{\mu \nu}^{T T}+\left(q_{\mu} q_{\nu}-q^{2} \eta_{\mu \nu}\right) \Theta^{S}$. Of course, if spurious polarizations decouple, they do so separately in the $T T$ and $S$ parts of matrix element (48); therefore, we can assume as well that it is traceless. In this case, the most general condition for decoupling is eq. (37), again with $\Delta_{\mu \nu}(q)$ analytic at $q=0$.

Substituting the spurious polarizations (47) into eq. (48) and equating all algebraically independent terms in the latter to $q^{2} \Delta_{\mu \nu}(q)$, we get several constraints on the small- $q$ behavior of the coefficients $A^{k}, \ldots, F^{k}$ and $\alpha^{k}, . ., \gamma^{k}$. In particular, terms proportional to $p_{\mu} p_{\nu} \bar{V}^{k} \epsilon^{k}$ give the condition

$$
C^{k}-(k+1) A^{k+1}-(s-k) \frac{q^{2}}{2} A^{k}=\mathcal{O}\left(q^{2}\right), \quad k=0, . ., s-1 .
$$

Terms proportional to $p_{(\mu} \bar{V}^{k-1} \epsilon_{\nu)}^{k}$ give

$$
(k+1) C^{k+1}-(s-k-1) \frac{q^{2}}{2} C^{k}+E^{k}=\mathcal{O}\left(q^{2}\right), \quad k=0, \ldots, s-2 .
$$

Finally, terms proportional to $\bar{V}_{\mu \nu}^{k+1} \epsilon^{k-1}$ give

$$
(k+1) E^{k+1}+(s-k) \frac{q^{2}}{2} E^{k}=\mathcal{O}\left(q^{2}\right), \quad k=0, \ldots, s-3 .
$$

Notice that we need $s \geq 3$ to obtain this full set of equations.

As in the Fermionic case, the coefficients $A^{k}, . ., F^{k}$ can be singular in the $q \rightarrow 0$ limit, but they must diverge less than $1 / q^{2}$. In this case eq. (56) implies

$$
\lim _{q \rightarrow 0} E^{s-2}(q)=0 .
$$


The vanishing of $E^{s-2}$ in the soft limit $q \rightarrow 0$ and eq. (55) then imply

$$
\lim _{q \rightarrow 0} C^{s-1}(q)=0
$$

Substituting this last equation into (154) we arrive at the main result of this subsection:

$$
\lim _{q \rightarrow 0} A^{s}(q)=0
$$

The vanishing of $A^{s}$ at zero graviton momentum is in contradiction with the equivalence principle, which demands $\lim _{q \rightarrow 0} A^{s}(q)=1$. So, massless Bosons of spin $s \geq 3$ cannot couple with gravity. It is straightforward to check that the set of equations (48, [55) has a solution satisfying the correct soft limit dictated by the principle of equivalence for $s=2$. This is possible thanks to the fact that for $s=2$ there is one less constraint to satisfy, namely eq. (156).

\section{Discussion and Conclusions}

In this paper, we borrowed ideas from the Weinberg-Witten no go theorem [3] as well as from known results on inconsistencies of gravitational coupling of high-spin massless particles, specifically from ref. [4], to show that no massless high-spin particle can be consistently coupled to gravity in flat space. The theorem exploited a particular onegraviton matrix element, whose form is constrained in the soft-graviton limit by the equivalence principle. We showed that, under fairly general assumptions, this constraint is incompatible with the decoupling of the spurious polarizations that one must necessarily introduce to write down the matrix element in a Lorentz covariant form.

The proof of the theorem was straightforward but not stunningly elegant. Clumsiness was the price we paid to allow for some mild non-locality in the matrix element. In particular, we did not demand analyticity at $q^{2}=0$ for the coefficients in our matrixelement expansion eqs. (25) or (48). Had we done so, we could have extended the matrix element to complex values of the momenta and put the graviton too on-shell, since the condition $p^{2}=q^{2}=(p+q)^{2}=0$ does have nontrivial complex solutions.

\section{No-Go in the BCFW Construction}

Analyticity for complex momenta is one of the main ingredients in the BCFW construction of S-matrix tree-level amplitudes [20]. The use of complex momenta 7 not only allows us

\footnotetext{
${ }^{7}$ Or equivalently the use of a space-time(s) metric of signature $(2,2)$.
} 
to write non-vanishing three-particle on-shell vertices, but it also allows us to deform two of the momenta in an arbitrary scattering amplitude along a special complex direction according to the formula

$$
p_{1} \rightarrow p_{1}+z q, \quad p_{2} \rightarrow p_{2}-z q, \quad p_{i}^{2}=p_{i} q=q^{2}=0, \quad i=1,2 .
$$

Any tree-level amplitude now becomes a rational function of the complex parameter $z$, with at most simple poles [20]. So, if a particular amplitude vanishes at large $z$, then it can be computed by knowing the position of the poles and the value of the residues. These are on-shell data that are completely specified by the three-point on-shell vertices. By applying the BCFW construction to a four-particle amplitude involving the exchange of a graviton, Benincasa and Cachazo [21] proved in an elegant manner that the only massless particles of spin $s>1$ that can be coupled to gravity are the graviton and the gravitino [21], and that they interact exactly as in supergravity. The most restrictive assumption in their construction is precisely the vanishing of the amplitude at large $z$. This property is far from obvious. It requires extra assumptions on the theory under consideration, in addition to Lorentz and gauge (or diffeomorphism) invariance [22]. We chose instead to keep our argument general even at the price of weakening our result.

One notable weakness of our argument is that it does not forbid the existence of more than one graviton; convincing arguments against this possibility have been given in the literature [18, 23]. Its main strength is that it does not rely on a particular field parametrization or on assuming a specific Lagrangian realization of the high-spin particle, since the only off-shell particle in the matrix element $\left\langle v, p+q\left|T_{\mu \nu}\right| u, p\right\rangle$ is the graviton itself.

Our theorem does rely on one property of the graviton: its masslessness. If the graviton were massive, or if its propagator were modified in the infrared -as in [19] for instancethen our theorem would not obtain. That alone is not sufficient to make gravitational interactions of high-spin massless fields consistent. Indeed, if the graviton was massive, one could integrate it out to obtain an effective theory valid for momenta lower than the graviton mass. The integration would unavoidably result in four-particle interactions involving the high-spin states. No example of consistent interactions of this type exists for spin $s>2$. Indeed, theorems proving the opposite in fairly general cases have been already given in the literature [24, 25, 26].

Of course, massive particles of spin larger than two do exist and their gravitational interactions do obey the principle of equivalence. In the case of massive particles, spurious 
polarizations $\left(v_{s}\right)$ become indistinguishable from physical longitudinal polarizations $\left(v_{l}\right)$ at energies $E \gg m 8$

$$
v_{l}=\frac{1}{m} v_{s}+\mathcal{O}(m / E),
$$

with $v_{s}$ given by eq. (35) for Fermions or eq. (45) for Bosons. Instead of signaling an inconsistency of the theory, now the non-decoupling of $v_{s}$ signals the onset of a strong coupling regime, since the matrix elements depend on inverse powers of the mass. The same property ensures that the massless limit is singular, as announced in the Introduction. One could try to cure this pathology by modifying the matrix elements by terms that explicitly depend on inverse powers of $m$ [9, 10]. Such terms do cancel mass singularities in $\left\langle v, p+q\left|T_{\mu \nu}\right| u_{l}, p\right\rangle$ [9, 10] but they also introduce additional singularities in previously regular matrix elements; namely $T_{\mu \nu}$ matrix elements between transverse states, i.e. states of highest helicity $\pm s$.

Massless particles in Anti de Sitter space-time are to all purposes indistinguishable from very light massive particles. The physical reason is that the curvature radius of AdS, $R_{A d S}$, acts as an IR cutoff effectively decoupling particles with larger Compton wavelength. Technically, this can be seen in the absence of mass discontinuities in the $m \rightarrow 0$ limit of the massive propagator [9, 13, 14]. In accordance with this expectation and with the existence of interacting massive particles, theories of massless interacting high-spin particles have been proposed [2]. Also in accordance with our expectations is the fact that these theories become strongly interacting at $E \sim 1 / R_{A d S}$, i.e. at the lowest energy for which one can localize a particle within the AdS Hubble radius. What this means for the ultimate viability of such theories is yet to be properly understood.

\section{A Limit on the Abelian Gauge Coupling of High-Spin Particles}

Our proof is easily adapted to constrain the coupling of charged massless particles to $U(1)$ gauge fields. We derive here the constraints for Fermionic particles only, in order to spare the reader further tedium, and because this example already teaches us a most important lesson. The most general helicity-conserving matrix element of a $U(1)$ current between on-shell spin $s$ states is

$$
\left\langle v, p+q\left|J_{\mu}\right| u, p\right\rangle=\sum_{k=0}^{n} A^{k} \bar{v}^{k} \gamma_{\mu} u^{k}, \quad s=n+1 / 2 .
$$

\footnotetext{
${ }^{8}$ In renormalizable gauge theories this property is known as the Goldstone Equivalence Theorem 27 , 28 .
} 
This matrix element is automatically conserved because both $u$ and $v$ obey the massless Dirac equation. When the $U(1)$ gauge vector is massless, spurious polarizations decoupling requires

$$
\left\langle v, p+q\left|J_{\mu}\right| u_{s}, p\right\rangle=q^{2} \Delta_{\mu}(q)
$$

with $\Delta_{\mu}(q)$ analytic at $q^{2}=0$. Substitution of the spurious polarization (35) into eq. (62) and eq. (63) gives the condition

$$
A^{k+1}-(n-k) \frac{q^{2}}{2} A^{k}=\mathcal{O}\left(q^{2}\right), \quad k=0, \ldots, n-1 .
$$

The charge of the high-spin state is defined [3] by

$$
e=\lim _{q \rightarrow 0} A^{n}(q)
$$

Since all coefficients $A^{k}$ must be less singular than $q^{2}$, the decoupling condition eq. (64) implies $e=0$ for $n \geq 1$ i.e. spin $s \geq 3 / 2$. This result is in accordance with supergravity, which indeed allows massless gravitini to have dipole and higher-multipole interactions in flat space, but not nonzero $U(1)$ charges. Charged spin-3/2 fields require either a mass 9 or a cosmological constant [29]. The result obtained here is also weaker than our main result on gravitational coupling. Indeed, positivity of energy forbids the existence of a particle with no energy but gravitational multipole coupling. Neutral massless particles with dipole or multipole electromagnetic coupling are instead a rather mundane possibility. There is one final aspect of charged particle dynamics that is not captured by our analysis. Standard renormalization group analysis says that Abelian interactions are free in the IR, so the IR charge of any massless particle is always zero. In a certain sense, our theorem rules out only a part of those theories that are already ruled out by the RG properties of unbroken Abelian gauge theories.

We would like to conclude with a speculation. It seems that "normal" massless particles can exist only for spin not larger than two. On the other hand, it could be possible that high-spin fields do not obey some of the most basic properties of "normal" particles. Could it be that they do not obey the principle of equivalence, yet they still interact with gravity through gravitational multipoles, as neutral particles can do when coupled to $U(1)$ fields? At the level of tri-linear interactions the answer is in the affirmative [30].

\footnotetext{
${ }^{9}$ Kaluza Klein gravitini have a charge proportional to their mass: $e \propto m / M_{P l}$.
} 
Yet, the cubic vertex of [30], or any other vertex that may have been proposed in the literature, cannot be extended beyond cubic order: Weinberg's theorem [5] forbids it 10 . This is seen by applying Weinberg's factorization argument, reviewed in Section 2, to a vertex with two spin $s$ particles and two gravitons. In the limit that one of the two gravitons becomes soft, eq. (5) implies that all other particles in the vertex must have the same gravitational charge, say $g_{g}=g_{s}=g_{s}^{\prime}=1$. This is true when the soft graviton ends on the external hard graviton. In this case the identity $g_{g}=1$ means simply that the graviton self-interacts in accordance with the principle of equivalence. On the other hand, when the soft graviton ends on either of the two spin $s$ lines, our general argument (and, of course, the explicit vertex in ref. [30]) gives $g_{s}=g_{s}^{\prime}=0$.

So, if high-spin massless fields do interact at all with "normal" matter, they cannot couple to any of its local degrees of freedom. They would have to couple to unusual, global degrees of freedom. This is not impossible since similar objects have already appeared in field theory. For instance singleton fields in AdS, which carry no bulk degree of freedom [31]; the graviton of 3-d gravity [32] and BF fields in various dimensions ([33] and references therein), which also propagate no local degrees of freedom, etc. Maybe highspin massless fields could constitute a new type of highly unusual, "quasi-topological" matter. Some positive hints that this may be true come from the study of the massless limit of Witten's open string field theory [34]

\section{Acknowledgments}

Work supported in part by NSF grants PHY-0245068 and PHY-0758032. Part of this work was also supported by a Marie Curie Excellence Chair, contract MEXC-CT-2003-509748 (SAG@SNS).

\section{References}

[1] L. Susskind, arXiv:hep-th/0302219.

[2] M. A. Vasiliev, Fortsch. Phys. 52, 702 (2004) arXiv:hep-th/0401177]; X. Bekaert, S. Cnockaert, C. Iazeolla and M. A. Vasiliev, arXiv:hep-th/0503128.

[3] S. Weinberg and E. Witten, Phys. Lett. B 96, 59 (1980).

[4] C. Aragone and S. Deser, Phys. Lett. B 86, 161 (1979).

\footnotetext{
${ }^{10}$ We thank A. Nicolis for pointing this out to us.
} 
[5] S. Weinberg, Phys. Rev. 135, B1049 (1964).

[6] M. T. Grisaru and H. N. Pendleton, Phys. Lett. B 67, 323 (1977).

[7] M. T. Grisaru, H. N. Pendleton and P. van Nieuwenhuizen, Phys. Rev. D 15, 996 (1977).

[8] G. Velo and D. Zwanziger, Phys. Rev. 186, 1337 (1969); Phys. Rev. 188, 2218 (1969);

G. Velo, Nucl. Phys. B 43, 389 (1972).

[9] M. Porrati, Phys. Lett. B 304, 77 (1993) arXiv:gr-qc/9301012.

[10] A. Cucchieri, M. Porrati and S. Deser, Phys. Rev. D 51, 4543 (1995) arXiv:hep-th/9408073.

[11] M. Porrati and R. Rahman, arXiv:0801.2581 [hep-th].

[12] M. Porrati, Phys. Lett. B 498, 92 (2001) arXiv:hep-th/0011152.

[13] P. A. Grassi and P. van Nieuwenhuizen, Phys. Lett. B 499, 174 (2001) arXiv:hep-th/0011278.

[14] S. Deser and A. Waldron, Phys. Lett. B 501, 134 (2001) arXiv:hep-th/0012014].

[15] D. Francia, J. Mourad and A. Sagnotti, arXiv:0803.3832 [hep-th].

[16] S. Deser and A. Waldron, arXiv:hep-th/0403059.

[17] S. Kamefuchi, L. O'Raifeartaigh and A. Salam, Nucl. Phys. 28, 529 (1961); J.S.R. Chisholm, Nucl. Phys. 26, 469 (1961).

[18] S. Weinberg, Phys. Rev. 138, B988 (1965).

[19] G. R. Dvali, G. Gabadadze and M. Porrati, Phys. Lett. B 485, 208 (2000) arXiv:hep-th/0005016.

[20] R. Britto, F. Cachazo and B. Feng, Nucl. Phys. B 715, 499 (2005) arXiv:hep-th/0412308; R. Britto, F. Cachazo, B. Feng and E. Witten, Phys. Rev. Lett. 94, 181602 (2005) arXiv:hep-th/0501052.

[21] P. Benincasa and F. Cachazo, arXiv:0705.4305 [hep-th].

[22] N. Arkani-Hamed and J. Kaplan, JHEP 0804, 076 (2008) arXiv:0801.2385 [hep-th]]. 
[23] N. Boulanger, T. Damour, L. Gualtieri and M. Henneaux, Nucl. Phys. B 597 (2001) 127 arXiv:hep-th/0007220; arXiv:hep-th/0009109.

[24] F. A. Berends, G. J. H. Burgers and H. van Dam, Nucl. Phys. B 260, 295 (1985).

[25] A. K. H. Bengtsson and I. Bengtsson, Class. Quant. Grav. 3, 927 (1986).

[26] X. Bekaert, N. Boulanger, S. Cnockaert and S. Leclercq, Fortsch. Phys. 54, 282 (2006) arXiv:hep-th/0602092.

[27] J. M. Cornwall, D. N. Levin and G. Tiktopoulos, Phys. Rev. D 10, 1145 (1974) [Erratum-ibid. D 11, 972 (1975)].

[28] M. S. Chanowitz and M. K. Gaillard, Nucl. Phys. B 261, 379 (1985).

[29] C. K. Zachos, Phys. Lett. B 76, 329 (1978).

[30] N. Boulanger and S. Leclercq, JHEP 0611, 034 (2006) arXiv:hep-th/0609221].

[31] P. A. M. Dirac, J. Math. Phys. 4, 901 (1963); E. Angelopoulos, M. Flato, C. Fronsdal and D. Sternheimer, Phys. Rev. D 23, 1278 (1981).

[32] E. Witten, Nucl. Phys. B 311, 46 (1988).

[33] D. Birmingham, M. Blau, M. Rakowski and G. Thompson, Phys. Rept. 209, 129 (1991).

[34] I. G. Koh and S. Ouvry, Phys. Lett. B 179, 115 (1986) [Erratum-ibid. 183B, 434 (1987)]; G. Bonelli, Nucl. Phys. B 669, 159 (2003) arXiv:hep-th/0305155]; A. Fotopoulos and M. Tsulaia, Phys. Rev. D 76, 025014 (2007) [arXiv:0705.2939 [hep-th]]. 